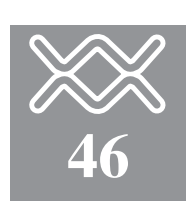

\title{
La nefrolitiasi: l'importanza del trattamento multidisciplinare
}

\author{
P. Rigatti ${ }^{1}$, P. Bellinzoni ${ }^{2}$ \\ ${ }^{1}$ Direttore Cattedra di Urologia \\ ${ }^{2}$ Dirigente Medico $I^{\circ}$ Livello
}

Università "Vita-Salute", Ospedale "San Raffaele", Milano

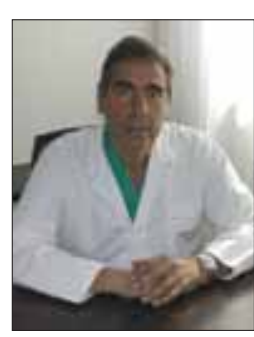

$\boldsymbol{I}$ $l$ trattamento della NL si è arricchito negli ultimi venti anni di procedure non invasive o semi-invasive. Ritenete che questo abbia costituito un progresso reale nell'approccio urologico alla malattia?

È noto che fino all'inizio degli anni ottanta il trattamento della nefrolitiasi era basato unicamente sulla terapia medica e sulla terapia chirurgica a cielo aperto.

Anche le indagini diagnostiche disponibili all'epoca, indubbiamente meno sofisticate e più invasive rispetto ad alcune attuali, quali l'ecografia utilizzata oggi anche per screening di massa, erano rivolte unicamente a pazienti sintomatici con situazioni spesso di una certa rilevanza clinica (calcolosi a stampo, calcolosi complesse, calcolosi ureterali ...).

Lo sviluppo quindi, nei primi anni ottanta, delle nuove procedure terapeutiche a bassa invasività quali la litotrissia extracorporea con onde d'urto
(ESWL) e la litolapassi endoscopica percutanea (PNL) e ureteroscopica (URS) con differenti fonti energetiche e l'utilizzo su ampia scala dell'ultrasonografia, hanno portato a un radicale cambiamento sia nell' inquadramento diagnostico che terapeutico della malattia litiasica.

Inoltre il progressivo miglioramento tecnologico delle apparecchiature per la litotrissia extracorporea con onde d'urto (ESWL), delle tecniche di approccio percutaneo (PNL) ed ureterorenoscopico (URS) nonché delle fonti energetiche necessarie alla litotrissia endoscopica, ha indubbiamente rappresentato un significativo progresso in campo urologico nel trattamento dell'urolitiasi.

Le molteplici esperienze riportate nell'ampia letteratura riguardante le nuove tecnologie mininvasive, consentono di attribuire il successo di tali metodiche a differenti aspetti quali la ridotta morbilità, la buona tolleranza dei pazienti in termini di minori tempi di ospedalizzazione con rapida ripresa dell'attività lavorativa e costi globali per la società inferiori.

La litotrissia extra- ed intracorporea rappresenta perciò un reale passo avanti nel trattamento della nefrolitiasi anche perché consente di affrontare e risolvere la maggior parte della patologia litiasica inclusa quella di modesta rilevanza fino ad allora destinata alla sola "osservazione" poichè la terapia chirurgica classica rappresentava, proprio per la sua invasività, un indubbio "over-treatment".

2. A vostro parere la disponibilità di queste procedure ha amplificato in modo inappropriato le indicazioni urologiche?

L'entusiasmo che ha inizialmente portato a un indiscriminato e talvolta inappropriato utilizzo delle nuove metodiche a bassa invasività, a scapito delle fino allora terapie tradizionali, ha consentito di sviluppare numerose e varie esperienze dalle quali si può oggi ottenere un inquadramento più chiaro dei criteri da seguire nella scelta del trattamento più idoneo ai differenti tipi di nefrolitiasi. L'intento di un buon Centro urologico deve essere quello di standardizzare le indicazioni terapeutiche fornendo al pa- 


\section{Giornale di Tecniche \\ Nefrologiche \\ \& Dialitiche \\ Anno XVI n ${ }^{\circ} 3$ \\ (C) Wichtig Editore 2004

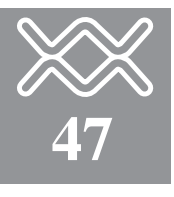

ziente delle chiare e corrette informazioni della sua problematica e delle possibilità di risoluzione della stessa in accordo comunque con le esigenze di ogni singolo paziente (compliance del paziente soprattutto in previsione di ripetuti trattamenti di ESWL).

La calcolosi urinaria può infatti avere molteplici aspetti in relazione prevalentemente alla sua localizzazione nelle cavità escretrici, alle dimensioni e alla composizione chimica dell'urolita nonché al quadro clinico associato (infezioni, stasi urinaria, insufficienza renale...). Quindi la possibilità che ha oggi l'urologo di poter fruire di un ampio ventaglio terapeutico rende indispensabile un corretto inquadramento diagnostico di ogni singolo caso al fine di evitare un uso inappropriato delle differenti metodiche con il rischio perciò di incorrere in spiacevoli sequele per il paziente.

Il paziente deve perciò esse informato oltre che della scelta terapeutica anche delle eventuali procedure aggiuntive necessarie o che si potrebbero rendere necessarie in conseguenza del trattamento effettuato, della possibilità di risoluzione in una o più sedute (trattamenti combinati: PNL+ESWL, Chirurgia a cielo aperto+ESWL, URS+ ESWL, o ESWL ripetute...) e di tutte le possibili complicanze correlate alle differenti terapie.

\section{La scelta della procedura è media- mente fatta in modo corretto nella maggior parte dei Centri urologici?}

La scelta della procedura terapeutica da parte dei differenti Centri urologici è talvolta influenzata dalla disponibilità e dalla familiarità nell'uso delle diverse metodiche che possono peraltro essere utilizzate sole o in associazione.

L'attuale vasta disponibilità di litotritori, consente di affrontare oggi la maggior parte dei casi di litiasi (calcoli renali $<2.5 \mathrm{~cm}$, calcoli ureterali lombari e pelvici $<1.5 \mathrm{~cm}$ ) con la litotrissia extracorporea con onde d'urto, tecnica scarsamente invasiva in grado di disgregare i calcoli in piccoli frammenti (mediante l'applicazione di onde d'urto generate all'esterno dell'or- ganismo) che vengono successivamente espulsi spontaneamente con le urine.

La principale sequela dell'ESWL è rappresentata dall'ostruzione ureterale da parte dei frammenti litiasici ottenuti dalla litotrissia stessa ed è tanto più frequente quanto maggiore è la massa litiasica trattata (calcoli $>1.5$ $\mathrm{cm})$. La possibilità di effettuare trattamenti accurati, mirati a frammentare parzialmente, anche se in più sedute, $i$ calcoli $>1.5 \mathrm{~cm}$, riduce talvolta il rischio ostruttivo della via escretrice. Anche il preventivo posizionamento di uno stent ureterale tipo DJ per calcoli $>2 \mathrm{~cm}$, per calcoli $>1.5 \mathrm{~cm}$ in monorene o in caso di idronefrosi ha lo scopo di salvaguardare il rene da fenomeni ostruttivi e dai conseguenti rischio infettivo e/o danno funzionale. Il DJ o la nefrostomia percutanea (PCN) devono essere posizionati successivamente all'ESWL nel caso in cui dovessero verificarsi le medesime situazioni ostruttive delle cavità escretrici o per intercorrenti quadri settici causati dai frammenti litiasici discesi in uretere, con lo scopo di drenare il rene e ripristinare la peristalsi ureterale indispensabile all'espulsione spontanea dei calcoli.

Da ciò emerge l'importanza della disponibilità e della conoscenza da parte di ogni urologo delle procedure di supporto alla litotrissia extra-corporea stessa.

La litotrissia intracorporea ureteroscopica (URS) e percutanea (PNL) consente la frammentazione dei calcoli, per contatto diretto tra urolita e fonte energetica, mediante l'utilizzo di strumenti endoscopici (ureterorenoscopio e nefoscopio). I frammenti ottenuti possono essere rimossi contestualmente all'atto operatorio con appositi strumenti o venire successivamente espulsi dal paziente spontaneamente con le urine.

Entrambi gli endoscopi a fibre ottiche sono dotati oltre che del canale di irrigazione, di canali operativi necessari al transito degli strumenti per la litotrissia (sonde a onde balistiche, sonde laser, sonde a ultrasuoni...) e per l'estrazione dei calcoli (pinze, cestelli...) in visione diretta.
L'ureterorenoscopia (URS) è indicata per il trattamento della maggior parte dei calcoli non suscettibili o refrattari all'ESWL e per la bonifica di persistenti frammenti litiasici ureterali esiti di ESWL. È una tecnica endoscopica minimamente invasiva poiché consente di esplorare l'uretere e le cavità renali con l'utilizzo di strumenti di piccolo calibro a fibre ottiche (rigidi o flessibili) introdotti principalmente per via retrograda attraverso l'uretra e la vescica e più raramente per via anterograda attraverso un preventivo mini-accesso percutaneo lombare.

La nefrolitotomia percutanea (PNL) è anch'essa una metodica mininvasiva che utilizza strumenti (rigidi o flessibili), di calibro lievemente superiore ai precedenti, che vengono introdotti direttamente nelle cavità renali attraverso un accesso percutaneo lombare di piccole dimensioni.

La PNL è indicata principalmente per calcoli renali di grosse dimensioni $>2.5 \mathrm{~cm}$ a stampo pielici e pielocaliciali, calcoli a stampo recidivi dopo chirurgia a cielo aperto, calcoli di maggiore consistenza (cistina e ossalato di calcio).

Ritengo però che un buon Centro urologico che disponga delle moderne apparecchiature a bassa invasività, nonché dell'adeguata esperienza nel loro utilizzo, non debba mai sottovalutare il giusto spazio da riservare alle "vecchie terapie" medica e chirurgica a cielo aperto, che per questo motivo non devono essere considerate tali, bensì complementari e/o aggiuntive alle moderne terapie a bassa invasività.

La terapia medica trova ancora oggi un suo giusto spazio, da sola o in associazione ad altre metodiche sia nell'intento di favorire l'espulsione spontanea di calcoli o di frammenti ottenuti dalla litotrissia che in quello di favorire la dissoluzione di alcuni tipi di uroliti mediante l'alcalinizzazione delle urine.

- L'espulsione spontanea è dovuta all'attività peristaltica dell'uretere ed è condizionata dalle dimensioni (il $70 \%$ dei calcoli $<5 \mathrm{~mm}$ e il $15 \%$ di quelli > $6 \mathrm{~mm}$ vengono espulsi spontaneamente) e dalla sede dei 
calcoli (calcoli $<6 \mathrm{~mm}$ dell'uretere lombare, iliaco e pelvico hanno rispettivamente il $12 \%, 22 \%$ e $45 \%$ di possibilità di espulsione spontanea): la somministrazione di una terapia antinfiammatoria ed alfa-litica spesso favorisce la naturale eliminazione spontanea degli uroliti.

- La dissoluzione dei calcoli di acido urico e di cistina avviene mediante la somministrazione orale di sostanze (citrati e bicarbonati di $\mathrm{Na}$ e K) in grado di alcalinizzare le urine $(\mathrm{pH}>6.5$ per acido urico e $\mathrm{pH}>7$ per cistina) e di sostanze quali l'allopurinolo (ipouricosurico), la Dpenicillamina e l'alfamercaptopropionil-glicina ecc. La dissoluzione della cistina, oggi usata solo in rari casi preceduti dalla litotrissia, può essere ottenuta mediante infusione diretta (percutanea o ureterale retrograda) nelle cavità escretrici di farmaci alcalinizzanti e favorenti la sua dissolubilità.

In casi di calcolosi di acido urico o di cistina particolarmente estesi, il preventivo utilizzo della litotrissia extrao intracorporea, facilita la dissoluzione chimica dei calcoli in conseguenza dell'aumentata superficie di contatto tra farmaco e frammenti litiasici derivata dalla frammentazione stessa.

Anche la terapia chirurgica classica a cielo aperto riveste tutt'oggi un suo ruolo, anche se notevolmente ridimensionato rispetto al passato, nell'approccio della calcolosi renale complessa in casi selezionati quali:

- anomalie anatomiche associate: stenosi giuntale o infundibolari, diverticoli caliciali...;

- calcoli voluminosi ramificati con estensione infundibulo-caliciali multiple;

- reni mai sottoposti a precedente chirurgia a cielo aperto.

Il successo della terapia chirurgica, all'epoca basato sulla completa bonifica dalla litiasi a scapito spesso della funzionalità renale, è attualmente inteso come totale frammentazione dei calcoli seguito, in tempi variabili, dall'eliminazione dei frammenti. Ciò è conseguentemente ottenibile con l'associazione della chirurgia alle nuove metodiche mininvasive (PNL, ESWL).
4. È percorribile secondo voi una gestione integrata del paziente fra nefrologo e urologo nell'ambito di un Centro di calcolosi renale? Ritenete davvero fattibile una gestione integrata, multidisciplinare, del paziente con $N L$ ?

L'approccio urologico al paziente affetto da NL è molto spesso esclusivamente causa-effetto. Infatti il paziente giunge alla nostra osservazione dopo un episodio acuto (colica renale, macroematuria...) o dopo episodi recidivanti di infezioni urinarie associate o meno a sintomatologia algica gravativa di modesta entità. Gli esami clinici spesso già in possesso del paziente al momento della visita o da noi consigliati (esami delle urine, ecografia renale, Rx addome smc, urografia...) svelano la presenza di una litiasi urinaria che è nostro compito indagare a fondo al fine di ottenere le informazioni necessarie per offrire al paziente la terapia meno invasiva e più idonea alla risoluzione della sua problematica.

A causa però di un'elevata tendenza alla recidivanza, variabile dal $26 \%$ al $52 \%$ a 10 anni come emerge dall' analisi della letteratura in merito, la terapia della calcolosi non deve avere come unico obiettivo l'eliminazione dell'urolita dalla via escretrice ma deve soprattutto mirare alla prevenzione della malattia. Per questo motivo la gestione integrata tra nefrologo ed urologo consente di offrire al paziente, attraverso un completo inquadramento della sua patologia, le principali norme preventive e terapeutiche necessarie alla risoluzione e al controllo della malattia litiasica.

5. Ritenete utile in termini di cost-efficacy uno studio metabolico del paziente con NL? Da chi dovrebbe essere fatto?

La tendenza alla recidiva varia a seconda del tipo di litiasi: per le calcolosi cistinica, urica e da infezione che mostrano una alta recidivanza a breve termine con conseguente maggiore rischio di indurre danni alla funzione renale, la terapia preventiva (idropinoterapia, terapia farmacologica e norme dietetiche) è indiscutibilmente necessaria. La calcolosi calcica mostra invece una grande variabilità nelle recidive e i trattamenti preventivi da adottare devono essere scelti in base al numero e all'intervallo delle recidive, all'età e alla familiarità dei soggetti .

Talvolta l'urolitiasi è l'espressione di malattie metaboliche misconosciute che devono essere diagnosticate e trattate non solo perché favorenti la nefrolitiasi, ma anche per altre possibili conseguenze cliniche.

Quindi una accurata valutazione da parte del nefrologo dei pazienti affetti da NL nell'ambito di un Centro di calcolosi renale consente di selezionare i soggetti da sottoporre allo studio metabolico al fine di ridurre i costi e di ottimizzare le risorse mediche nella gestione del paziente litiasico.

6. Esiste nella vostra realtà regionale la possibilità di accedere a un Centro per lo studio metabolico della NL?

L'esperienza maturata, negli ultimi 20 anni, dalla nostra Divisione di urologia nel trattamento della nefrolitiasi, basata essenzialmente sull'utilizzo delle differenti metodiche a bassa invasività (ESWL, URS, PNL), ma anche sulla terapia chirurgica convenzionale, ha richiamato nel nostro reparto un gran numero di pazienti affetti dai più svariati tipi di calcolosi urinaria. Ciò ha consentito di stabilire, nella gestione del paziente litiasico, una importante collaborazione con la Divisione di nefrologia del nostro Ospedale alla quale i pazienti possono essere indirizzati successivamente alla terapia da noi effettuata per essere accuratamente valutati presso il Centro per lo Studio Metabolico della Nefrolitiasi al fine di completare l'inquadramento diagnostico e terapeutico dei pazienti "nefro-urologici". 